\title{
Assembly of the isomerized TFIIA-TFIID-TATA ternary complex is necessary and sufficient for gene activation
}

\author{
Tianhuai Chi and Michael Carey ${ }^{1}$ \\ Department of Biological Chemistry, University of California, Los Angeles, School of Medicine, \\ Los Angeles, California 90095-1737 USA
}

\begin{abstract}
The prevailing view of eukaryotic gene activation poses that activators stimulate transcription by recruiting limiting components of the general transcription machinery to a core promoter. In one such model case, activation by the Epstein-Barr virus ZEBRA protein correlated closely with recruitment of the general transcription factors TFIIA and TFIID (the DA complex) as measured by DNase I footprinting and gel mobility shift assays. We now report that simple recruitment is not sufficient for full-level activation. An additional concentration-independent, rate-limiting step is activator-mediated isomerization of the DA complex characterized by an extended TFIID footprint. The isomerized complex supports both binding of TFIIB in gel mobility shift assays and activated transcription in heat-treated nuclear extracts, even after removal of ZEBRA. Surprisingly, the regulatory phenomenon of synergy was manifested only when the concentration of TFIID was limiting. When the DA complex was saturating, transcription was not synergistic, as indicated by the ability of a single activator to induce isomerization effectively and turn on a gene. On the basis of these observations, we propose a new biochemical model for eukaryotic gene activation and synergy.
\end{abstract}

[Key Words: Epstein-Barr virus; ZEBRA protein; TFIIA-TFIID-TATA complex; gene activation]

Received June 28, 1996; revised version accepted August 28, 1996.

The question of how activators stimulate transcription has dominated the field of eukaryotic gene regulation throughout the last decade. The complexity of the problem is emphasized by the paradoxically simple organization of upstream activator proteins and the large size and complexity of the RNA polymerase II (pol II) transcription machinery. The details of how activators contact the transcription machinery and the consequences of such interactions have provided a multidisciplinary challenge encompassing both biochemistry and genetics. Historically, the issue was first addressed by biochemical studies in mammalian systems, where Roeder and colleagues showed that basal and activated transcription could be reconstituted in vitro using pol II, a series of general transcription factors, ultimately resolved into six distinct proteins termed TFIIA, TFIIB, TFIID, TFIIE, TFIIF, and TFIIH, and a group of coactivator proteins collectively called the USA fraction (Hori and Carey 1994; Tjian and Maniatis 1994; Zawel and Reinberg 1995; Goodrich et al. 1996).

According to one model, the general factors assemble into a $2500-\mathrm{kD}$ preinitiation complex in a stepwise man-

${ }^{1}$ Corresponding author. ner (Zawel and Reinberg 1995), nucleated by the binding of TFIIA and TFIID to the core promoter and culminating with the association of TFIIH and subsequent ATPdependent opening of the start site (Wang et al. 1992a; Holstege et al. 1996). An alternative model poses that either a complete set or subset of the factors is assembled into a holoenzyme, which can be recruited to the promoter in a single step (Koleske and Young 1994; Barberis et al. 1995; Carey 1995; Koleske and Young 1995; Ossipow et al. 1995; Zawel and Reinberg 1995; Chao et al. 1996; Maldonado et al. 1996). Activators accelerate limiting steps in assembly of an intact transcription complex by both the holoenzyme and stepwise models.

Current biochemical data support the idea that recruitment of TFIID in combination with TFIIA is an important activator-targeted step. For example, kinetic experiments on transcription and open complex assembly with model activators, including the nonacidic EpsteinBarr virus (EBV) trans-activator ZEBRA and the GAL4derived acidic activators GAL4-AH and GAL4-VP16, have shown that preassembly of the subcomplex containing TFIID and TFIIA ("the DA complex") with activator bypasses a rate-limiting step in gene activation (Wang et al. 1992; White et al. 1992; Chi and Carey 1993). DNase I footprinting and gel shift studies con- 
firmed that GAL4-VP16 and ZEBRA enhance binding of DA to the core promoter (Lieberman and Berk 1994; Chi et al. 1995; Kobayashi et al. 1995). Related studies have shown that the coactivators PC4 and high mobility group 2 (HMG2) also act at the DA step, although it is not clear whether they function in recruitment per se (Ge and Roeder 1994; Kaiser et al. 1995; Shykind et al. 1995).

Consistent with the aforementioned view is the observation that synergy, the greater-than-additive effect of multiple activators on transcription and the molecular basis of combinatorial control, is first manifested during formation of the DA (Chi et al. 1995) or D complex (Sauer et al. 1995) when the TFIID concentration and, hence, complex assembly is limiting. In the case of ZEBRA, the magnitude of the recruitment in DNase I footprinting and gel mobility-shift assays parallels the synergistic effect of ZEBRA on transcription. GAL4 VP16 also targets the DA complex synergistically, albeit less effectively than ZEBRA (Chi et al. 1995). Taken together the recruitment of the DA subcomplex can be viewed as a key checkpoint in gene activation, an idea reinforced by recent in vivo studies in yeast /Chatterjee and Struhl 1995; Klages and Strubin 1995; Stargell and Struhl 1995; Xiao et al. 1995). This observation, however, has been difficult to reconcile with the purported role of TFIIB in gene activation. Both ZEBRA and the GAL4-derived acidic activators can recruit TFIIB to a promoter under certain experimental conditions, an issue we address herein (Lin and Green 1991; Lin et al. 1991; Lieberman and Berk 1994; Chi et al. 1995).

The goal of the present study was to determine whether recruitment of the DA complex was sufficient for gene activation in vitro, a premise supported by our previous biochemical studies. We hypothesized that if the physiological goal of ZEBRA was to recruit DA to the promoter, where it bound TFIIB or perhaps the holoenzyme, this would predict that saturation of a core promoter with DA should bypass the requirement for ZEBRA. However, this prediction was not borne out in footprinting and transcription assays with pure TFIIA and TFIID. Instead, we found that a concentration-independent, rate-limiting step is an activator-mediated isomerization of the DA complex. This isomerized DA complex is necessary and sufficient for binding of TFIIB and the remaining general transcription factors, even after removal of ZEBRA. The experimental approach that led to this finding also revealed that the phenomenon of synergy and the activator-mediated enhancement of TFIIB binding are regulatory events that are only manifested in vitro, on naked DNA templates, when the concentration of TFIID is limiting.

\section{Results \\ ZEBRA induces an isomerization of the TFIID-TFIIA-TATA ternary complex}

Figure 1 illustrates that ZEBRA induces a stable conformational change in, or isomerization of, the TFIIA-
TFIID-TATA ternary complex (the DA complex) as measured by a DNase I footprinting assay performed on a model reporter template bearing seven ZEBRA sites upstream of the minimal adenovirus E4 core promoter $\left(\mathrm{Z}_{7} \mathrm{E} 4 \mathrm{~T}\right)$. At a saturating concentration of TFIID and TFIIA (DA) a 27-bp DNase I footprint is generated encompassing the TATA box and surrounding nucleotides (lane 3). Addition of ZEBRA induces an isomerization event, resulting in the appearance of a $>50$-bp extended footprint characterized by a closely cropped set of enhanced bands between the ZEBRA sites and the TATA (lane 4; see arrows) and an alternating set of downstream enhancements (arrows) and protections (brackets). The extended footprint is stable because removal of the activator from the template by addition of a competing oligonucleotide, bearing a high-affinity ZEBRA site, abolishes the ZEBRA footprint (cf. lanes 5 and 7 with lanes 2

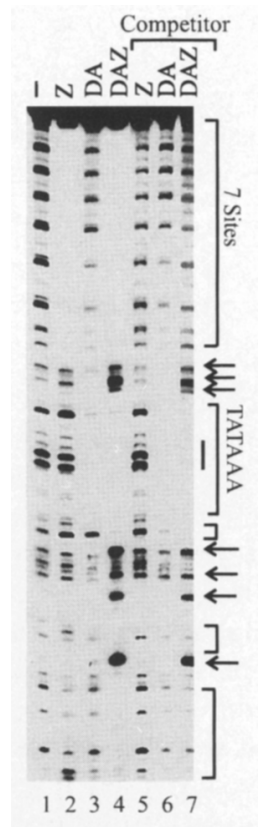

Figure 1. ZEBRA is required for inducing, but not maintaining, isomerization of the DA complex. A ${ }^{32} \mathrm{P}$-labeled DNA fragment bearing seven high-affinity ZEBRA-binding sites upstream of the adenovirus $E 4$ core promoter $\left(Z_{7} E 4 T\right)$ was incubated with recombinant ZEBRA $(Z)$, template saturating concentrations (500 ng) of TFIID (D) and recombinant TFIIA (A). After a 30-min preincubation at $30^{\circ} \mathrm{C}$ an 800 -fold molar excess of a competitor oligonucleotide was added as indicated; the oligonucleotide bore a high-affinity ZEBRA-binding site (Carey et al. 1992). After $60 \mathrm{~min}$ the mixtures were subjected to DNase I footprinting analysis and the digestion products were fractionated on a $6 \%$ polyacrylamide/urea sequencing gel. The brackets indicate protected regions and the arrows are enhancements. The black rectangle denotes the location of the TATA box. The autoradiographs shown above and in subsequent figures were scanned into Adobe Photoshop 3.0 using a ScanMaker III (Microtek) imported into Corel Draw 5.0 and labeled. The figures were printed onto glossy paper using a Tektronix dye sublimation printer. In some cases nonessential lanes from the original autoradiograph were excised and omitted. 
and 4) but has little effect on the extended DA footprint (cf. lanes 4 and 7). The isomerization is not attributable to contaminants because the TFIID used here is a highly fractionated, immunopurified protein (Zhou et al. 1992; Lieberman and Berk 1994). Taken together with our previous study, the data suggest that highly inducible gene activation might be a combination of recruitment and isomerization of the DA complex. We will test this hypothesis below.

The "extended footprint" was first discovered by Roeder and colleagues using crude preparations of TFIID and was interpreted then as a conformational change in the TFIID complex thought to be important for activation (Horikoshi et al. 1988a). More recent studies have indicated that the extension is attributable to TBP-associated factors (TAFs) constituting the intact TFIID protein; TAFs have been shown, much like the USA coactivator proteins, to be essential for activated transcription (Tjian and Maniatis 1994). Despite this initial observation, the significance of the footprint has been difficult to establish without pure TFIID and efficient systems to measure its activity. By using homogenous TFIIA and TFIID and measuring transcription in an efficient in vitro system we will demonstrate that the conformational change is indeed a rate-limiting step in gene activation in vitro.

\section{The isomerized DA complex is sufficient for gene activation}

To assess promoter occupancy and transcription by the DA complex under identical conditions, both the footprinting and transcription experiments were performed in parallel on the same ${ }^{32} \mathrm{P}$-labeled DNA template. This approach, although technically difficult, is essential to compare accurately the physical status of the complex with its transcriptional activity. Each set of experiments described below was performed multiple times and a typical example is shown.

Figure 2 shows that the isomerization event described above is limiting for activated levels of transcription. Thus, ZEBRA is necessary to induce DA isomerization. However, once isomerization occurs, ZEBRA can be removed by addition of a competitor oligonucleotide and the modified DA complex is sufficient to support activated levels of transcription.

A saturating amount of DA, as measured by DNase I footprinting on naked DNA, was incubated with a ${ }^{32} \mathrm{P}$ labeled $\mathrm{Z}_{7} \mathrm{E} 4 \mathrm{~T}$ promoter fragment in either the presence or absence of ZEBRA. Excess competitor oligonucleotide was added either immediately, at time 0 (lanes 3,4 ), before ZEBRA could induce the isomerization, or after 30 min (lanes 5,6), following the appearance of the extended footprint. After an additional $15 \mathrm{~min}$, the resulting complexes were incubated in a heat-treated HeLa nuclear extract and transcription was measured by a run-off assay in the presence of $\left[\alpha_{-}^{-32} \mathrm{P}\right]$ UTP. Heat treatment has been shown to inactivate TFIID selectively (Nakajima et al. 1988) and the TFIID-inactivated extract serves as a convenient and highly active system to measure the transcriptional activity of the DA subcomplex. It is significantly, by an order of magnitude, more active than our reconstituted transcription system comprising all pure transcription components. Unlike the extract, the pure system is ineffective in mediating response to upstream activators.

In the absence of competitor, ZEBRA stimulates transcription strongly (lanes 1,2). Figure 2A shows that when competitor was added at $30 \mathrm{~min}$, after DA was allowed to isomerize, high level transcription was still observed (cf. lane 2 with lane 6) although, as we will show in the footprints below, ZEBRA had been competed away. It was unlikely that the amount of oligonucleotide was insufficient to compete ZEBRA effectively from the template because when it was added at time 0 , before ZEBRA had the opportunity to induce isomerization, no activation was observed (cf. lanes 2 and 4). This latter control also eliminates the possibility that ZEBRA, when bound to the oligonucleotide, could somehow activate transcription. The effect is not core promoter-specific as it is also observed on $\mathrm{Z}_{7} \mathrm{M}$ (Chi et al. 1995), containing the core promoter from BMRF-1, a natural EBV ZEBRA-responsive promoter (lanes 7-10).

The DNase I footprints in Figure 2B validated the transcription results in Figure $2 \mathrm{~A}$ by confirming that the competitor oligonucleotide was capable of competing quantitatively ZEBRA from its sites in the presence of heat-treated nuclear extract. Comparison of lanes 4 and 7 , performed in the absence of extract, with lanes 11 and 14 , performed in the presence of extract, showed that the activator-induced extended footprint is relatively stable both before (lanes 4 and 11) and after (lanes 7 and 14) competition. The efficacy of the oligonucleotide in competing ZEBRA was further confirmed by gel shifts assays (data not shown).

Figure $2 \mathrm{~B}$ makes the additional critical point that saturation, or nearly so, of the TATA by DA is not sufficient to overcome the requirement for ZEBRA. Thus, densitometric analysis of the autoradiograph (the enhanced bands in the center of the TATA; black rectangle) confirms that the DA footprint in the extract represents almost complete $(85 \%)$ protection (cf. lanes 1 and 6 with lanes 8 and 13). The data in Figure 2, A and B, taken with our previous study, imply that a significant limiting step in gene activation is a combination of recruitment and isomerization of the DA complex.

To further eliminate the possibility that ZEBRA is still bound to the promoter in the presence of competitor, perhaps to an extent that cannot be detected by footprinting, we performed an additional control experiment. Immediately after isomerization, the ZEBRAbinding sites in $\mathrm{Z}_{7} \mathrm{E} 4 \mathrm{~T}$ were separated from the core promoter by cleavage with the restriction endonuclease $X b a I$. Complete cleavage was confirmed by the observation that the radiolabeled DNA template shifted from a slower to a faster mobility form in the gel after endonuclease treatment. Figure $2 \mathrm{C}$ shows that after ZEBRAmediated isomerization, the DA complex was still able to support activated levels of transcription in the absence of the upstream ZEBRA sites (cf. lanes 1 and 2 with 


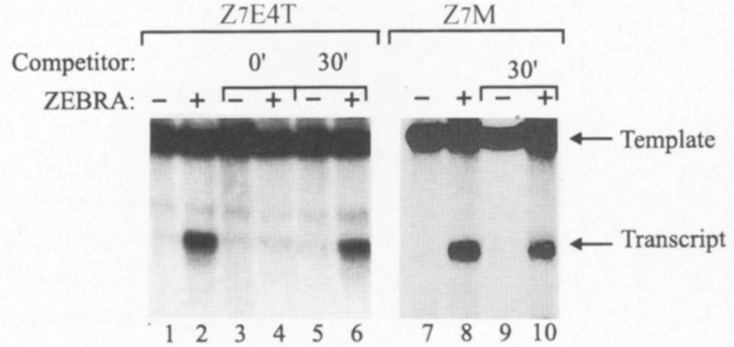

B

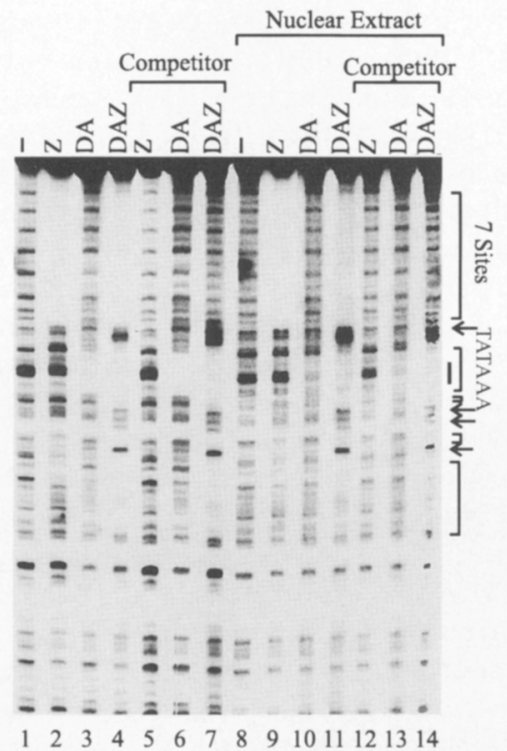

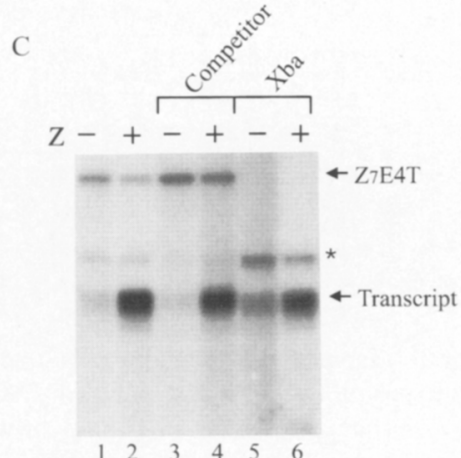

$\mathrm{D}$

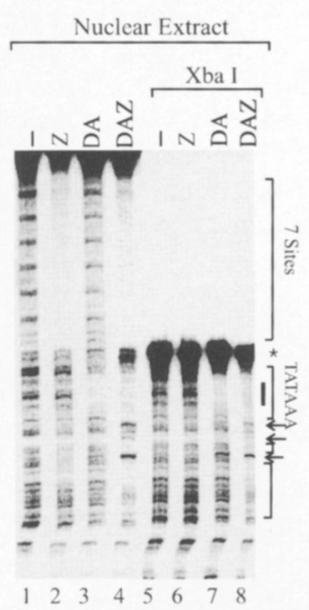

Figure 2. ZEBRA becomes dispensable for high-level transcription after DA isomerization. Oligonucleotide competition of ZEBRA $(A, B)$. $(A)$ Transcription. The ${ }^{32} \mathrm{P}$-labeled template either $\mathrm{Z}_{7} \mathrm{E} 4 \mathrm{~T}$ or $\mathrm{Z}_{7} \mathrm{M}$ was preincubated with saturating DAZ for 30 min in the absence or presence of the competitor oligonucleotide (Competitor), which in the case of $Z_{7} E 4 T$ was added either at the beginning $\left(0^{\prime}\right)$ or at the end $\left(30^{\prime}\right)$ of the preincubation and in the case of $Z_{7} M$, only at the end. After 15 min, the complexes were incubated in heat-treated HeLa cell nuclear extracts. After an additional $15 \mathrm{~min}$, the complexes were assayed for their abilities to support transcription by supplementing the reactions with $\left[\alpha{ }^{-32} \mathrm{P}\right]$ UTP, GTP, CTP, and ATP. After a 20 -min transcription reaction the radiolabeled transcripts were isolated and resolved on an $8 \%$ polyacrylamide/urea gel. The templates and the run-off transcripts are marked by arrows. $(B)$ Footprinting. The reactions were otherwise identical except nucleotides were omitted, DNase I was added and the resulting footprints were resolved on a $4 \%$ sequencing gel. The footprints performed in the extract are smearier when cornpared with the pure factors as a result of endogenous nucleases. Using restriction enzyme cleavage as a means to remove ZEBRA $(C, D)$. The procedures are identical to those described in $A$ and $B$ except that after a 30 -min preincubation, 10 units of $X b a I$ were added to the reaction as indicated. After a 30-min digestion, heat-treated nuclear extracts were added and transcription or DNase I footprinting assays were performed as in $A$ and $B$. Note the corresponding decrease in mobility of the ${ }^{32}$ P-labeled DNA template $\left({ }^{*}\right)$ in both the transcription assays $(C)$ and the footprints $(D)$. Also note the stability of the extended footprint in the absence of ZEBRA (lane 8 ). Cleavage apparently raises the basal transcription level slightly and induces a correspondingly slight enhancement of downstream bands in the footprint.

lanes 5 and 6). Note, however, that the extent of activation was slightly diminished because of an increase in basal transcription on the cleaved templates. Figure $2 \mathrm{D}$ confirms that ZEBRA induces a stable extended footprint on these cleaved fragments. The data in Figure 2, A and B, imply that the assembly of the isomerized DA complex is necessary and sufficient for activated transcription and once assembled ZEBRA is dispensable.

\section{Transcriptional activation is at the level of initiation}

The enhanced transcription is attributable to enhanced pol II initiation complex assembly as measured by the formation of open complexes in the heat-treated nuclear extracts. Open complex formation can be monitored by potassium permanganate probing, which modifies thymidines in single-stranded DNA (Wang et al. 1992a). The modified thymidines can be detected by a primer extension assay with Taq DNA polymerase, which stalls at the modified residues. Figure 3 shows that the enhanced transcription in Figure 2 correlates with an increase in the permanganate sensitivity of the six thymidine residues (bracket) encompassing the start site of our $\mathrm{Z}_{7} \mathrm{E} 4 \mathrm{~T}$ reporter template (cf. lane 4 with lane 5). ZEBRA could be removed without affecting the extent or timing of open complex formation once the DA complex had been assembled (cf. lanes 1-4 with lanes 6-9). 


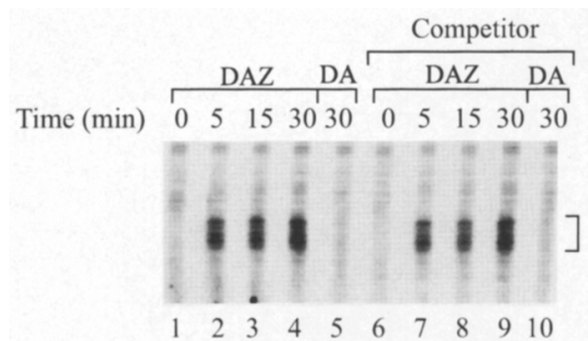

Figure 3. The effect of oligonucleotide challenge on open complex assembly. $Z_{7} E 4 T$ was preincubated with DA or DAZ for 30 min before addition of the competitor oligonucleotide (lanes 6-10). After $15 \mathrm{~min}$, the reactions were complemented with heat-treated nuclear extract, $\alpha$-amanitin $(2 \mathrm{ng} / \mu \mathrm{l})$ and dATP $(0.5$ $\mathrm{mm})$. At the indicated times thereafter $(0-30 \mathrm{~min}), 6 \mathrm{~mm}$ potassium permanganate was added. After an additional $4 \mathrm{~min}$, the modification reaction was terminated with 2-mercaptoethanol and the DNA was subjected to primer extension analysis. Taq DNA polymerase stalls at the modified $T$ residues and the stall products (bracket), a measure of open complex formation, are resolved on $6 \%$ polyacrylamide/urea gels. An autoradiograph of the gel is shown.

Recruitment of TFIIB to the isomerized DA complex is independent of ZEBRA

According to the stepwise model for transcription complex assembly, TFIIB binding follows formation of the DA complex. Our observation that the isomerized DA complex suffices to support efficient transcription predicts that once the DA complex is formed, TFIIB can associate spontaneously with it (DAB complex). Figure $4 \mathrm{~A}$ tests this prediction using magnesium agarose gel shift analysis of $\mathrm{DAB}$ complexes formed in the presence and absence of ZEBRA.

Magnesium agarose gels were used previously to resolve the DA complex and monitor binding of TFIIB /Chi et al. 1995). These studies revealed that TFIIB can associate with and promote the formation of the DAZ complex when TFIID is not saturating (DABZ complex). Although a supershift of the DAZ complex to the DABZ complex can often be observed, the major effect is stabilization or enhanced formation of the complex (Chi et al. 1995). Using the enhanced complex formation as a measure of TFIIB binding, we now show that TFIIB can associate stably with the isomerized DA complex even after removal ofZEBRA.

The DA complex was first assembled for $60 \mathrm{~min}$ on a radiolabeled $\mathrm{Z}_{7} \mathrm{M}$ probe in the presence and absence of ZEBRA. Increasing amounts of TFIIB were then added to the reaction for $15 \mathrm{~min}$ either before (lanes 13-18) or after (lanes 7-12) addition of competitor oligonucleotide. The final components of the two sets of reactions are identical, but in the second set TFIIB was subjected to ZEBRA action before oligonucleotide competition (lanes 16-18). The complexes were then visualized by gel shift at the indicated final time points $(90,150$, or $210 \mathrm{~min})$. In the absence of competitor oligonucleotide, the ZEBRAstimulated DA complex increased with time, reaching a plateau at $210 \mathrm{~min}$ after the initial incubation (cf. lane 3 with lane 4). In contrast, once ZEBRA was removed, the DA complex decayed with a half-life of $1 \mathrm{hr}$ (lane 6). Addition of high concentrations of TFIIB either before or after ZEBRA removal stabilized the DA complex such that the half-life increased to $2 \mathrm{hr}$ (cf. lane 6 with lanes 10 and 16). In contrast, TFIIB had only a minor effect on the small amount of basal DA complex formed in the absence of ZEBRA (lanes $3,7,13)$ and this effect was only observed with high TFIIB concentrations at later time points. We conclude that the isomerization of the DA complex is necessary and essentially sufficient for efficient TFIIB assembly as ZEBRA has only a marginal effect on TFIIB binding once the DA complex is preassembled (cf. lanes 10-12 with 16-18).

Paradoxically, a direct activator-TFIIB interaction, identified by affinity chromatography, is generally thought to be important for TFIIB recruitment and synergistic transcription stimulation by diverse activators including ZEBRA (Sundseth and Hansen 1992; Wang et al. 1992b; Chi and Carey 1993; Choy and Green 1993; Roberts et al. 1993, 1995; Kim and Roeder 1994). We reasoned that although TFIIB can bind to the preassembled DA complex spontaneously, a ZEBRA:TFIIB interaction observed previously (Chi et al. 1995) might affect cooperative assembly of the DAB complex when TFIII) and TFIIA are limiting and thus influence synergistic transcription complex assembly.

To address this hypothesis, we tested the effects of TFIIB on DA complex assembly on templates bearing one, three, and seven sites $\left(\mathrm{Z}_{1,3,7} \mathrm{M}\right)$ at limiting TFIID concentrations (Fig. 4B); transcription on templates bearing three and seven sites is highly synergistic when compared with a template containing a single site. As we observed previously (Chi et al. 1995), ZEBRA stimulated DA complex assembly (DAZ) strongly on $Z_{7} M$ (lanes 13,14 ), moderately on $Z_{3} M$ (lanes 8,9 ), but was totally inactive above the basal level on $Z_{1} M$ (lanes 3,4). These data exemplify the site-dependent synergistic effect of ZEBRA on recruitment of the DA complex as we reported previously (Chi et al. 1995). The presence of TFIIB, however, moderately $(\sim 2 \times)$ enhanced complex assembly on $Z_{3} M($ lanes 9,10$)$, but had no effect on $Z_{1} M$. The effect was not as evident on $Z_{7} M$ because DA complex assembly was saturating under the conditions used. However, we have shown previously that at shorter time points a stabilizing effect of TFIIB on DAZ complex assembly on $\mathrm{Z}_{7} \mathrm{M}$ can be observed (see Fig. $2 \mathrm{~B}$ in Chi et al. 1995). Because TFIIB had no effect on $Z_{1} M$, the cooperative assembly of the $D A B$ complex on $Z_{3} M$ enhances synergistic transcription. We conclude that when DA complex assembly is limiting, the ZEBRA-TFIIB interaction may play a role, albeit small, in TFIIB recruitment, DAB complex assembly, and transcriptional synergy.

The stimulatory effect of TFIIB on DA complex assembly can be observed in an in vitro transcription reaction. Figure 4C shows an example of such an experiment performed on $\mathrm{Z}_{7} \mathrm{E} 4 \mathrm{~T}$ in a heat-treated, TFIIB-immunodepleted extract. Preincubation of TFIIB with limiting concentrations of DA and ZEBRA for a short time period 
A

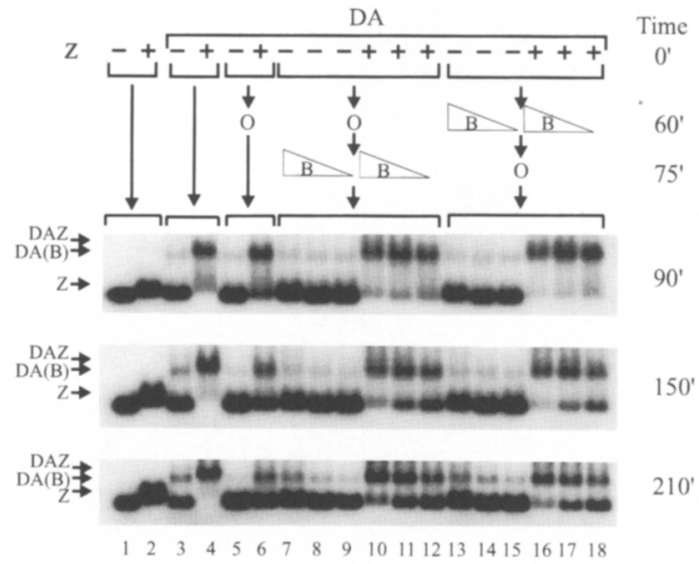

B

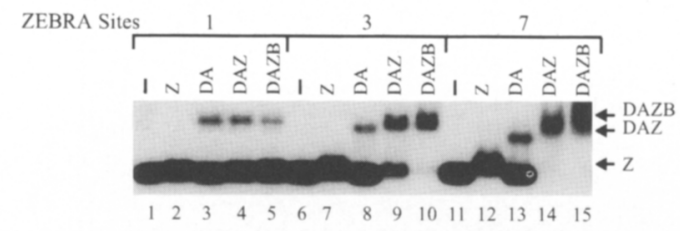

$\mathrm{C}$

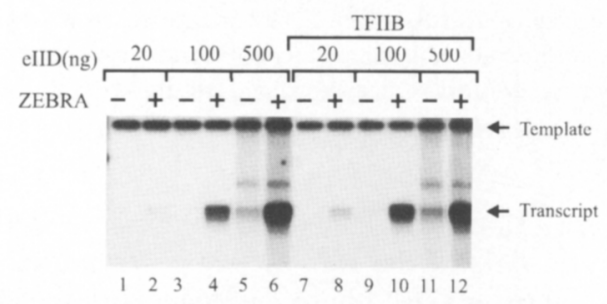

Figure 4. TFIIB recruitment. (A) A gel mobility shift assay revealing efficient association of TFIIB with the isomerized DA complex in the absence of ZEBRA. The probe $\mathrm{Z}_{7} \mathrm{M}$ was incubated with DA (200 ng of TFIID) in the absence $(-)$ or presence $1+1$ of ZEBRA (Z) beginning at time $0 \mathrm{~min}$ as indicated to the right (lanes 3-18). After $60 \mathrm{~min}$, competitor oligonucleotide (O) was added to one set of reactions (lane 7-12) for $15 \mathrm{~min}$ to remove ZEBRA before addition, at $75 \mathrm{~min}$, of varying amounts of recombinant TFIIB $(150,30,6 \mathrm{ng}$; lanes $7-9$ and $10-12$, respectively); the association of TFIIB with the DA complex take place in the absence of ZEBRA in this set of reactions. The complexes were then fractionated on a $1.4 \% \mathrm{Mg}$-agarose gel 90 , 150 , or $210 \mathrm{~min}$ after the initial incubation, or 15,60 , or $120 \mathrm{~min}$ after TFIIB addition, to reveal the extent of the DAB complex assembly and its decay over time. In another set of reactions (lanes 13-18), TFIIB was added 15 min before the competitor to allow ZEBRA to interact directly with TFIIB during DAB complex assembly. The complexes are then assayed similarly as above to reveal the consequence of TFIIB-ZEBRA interaction on $\mathrm{DAB}$ complex formation. As controls, nothing (lanes 3,4) or only the competitor $(\mathrm{O})$ (lanes 5,6$)$ was added to the reaction. The position of various complexes are indicated to the left of the panel. $(B)$ A gel shift assay indicating synergistic assembly of DABZ complexes when DA is limiting. $Z_{1,3,7}-\mathrm{M}$ were incubated with various combinations of ZEBRA (Z), TFIIA (A), $200 \mathrm{ng}$ TFIID (D), $160 \mathrm{ng}$ recombinant TFIIB (B) as indicated for $1 \mathrm{hr}$ before gel electrophoresis. The positions of various complexes are marked to the right of the panel. $(C)$ A run-off transcription assay demonstrating the effect of TFIIB on transcription. The $\mathrm{Z}_{7} \mathrm{E} 4 \mathrm{~T}$ template was preincubated with TFIIA and increasing concentrations of TFID $(20,100,500 \mathrm{ng})$ in the absence or presence of $20 \mathrm{ng}$ of TFIIB. ZEBRA was also present where indicated. After $30 \mathrm{~min}$, the reactions were complemented with TFIIDand TFIIB-depletedHeLa nuclear extracts and nucleoside triphosphates; TFIIB was included where it was absent during the preincubations (lanes 1-6). After 25 min transcription was terminated and the products were fractionated on a $6 \%$ polyacrylamide/urea gel.

(lanes 7-12) generated a reproducible twofold higher transcriptional response than when TFIIB was added after the DA preincubation (lanes 1-6). Transcriptional effects of similar magnitude have also been observed with TFIIB preincubation in other experiments and in previous studies (Wang et al. 1992b; Chi and Carey 1993).

\section{Synergistic gene activation is manifested when the TFIID concentration is limiting}

By comparing templates bearing three and seven ZEBRA sites with a template bearing a single site we found previously (see also Fig. 4B) that ZEBRA could recruit synergistically the DA complex to the TATA box when TFIID was made limiting (Chi et al. 1995). Furthermore, the magnitude of DA recruitment roughly paralleled the levels of gene activation and open complex formation in a HeLa nuclear extract (Chi and Carey 1993). However, the observation in Figure 2 that binding of DA alone was not sufficient for activation led us to address the question of whether the synergy could be uncoupled from recruitment of DA.

One hypothesis was that the multiple activators are required to both recruit DA synergistically and induce isomerization. An alternative posed that once DA was bound the energy required for isomerization is less than that required for recruitment, and hence fewer activators are necessary for activation. According to this latter hypothesis a single activator might be able to isomerize the DA complex and hence activate a gene.

Figure 5, A and B, distinguishes between these possibilities, showing that at limiting concentrations of TFIID (20 and $100 \mathrm{ng}$ ) transcription is synergistic when comparing templates bearing one and seven sites. However, at saturating concentrations of TFIID (500 ng), activated transcription and isomerization are no longer synergistic and can be mediated efficiently by a single activator.

The in vitro transcription experiment in Figure 5A shows that over a wide range of TFIID concentrations transcription was stimulated strongly on $\mathrm{Z}_{7} \mathrm{E} 4 \mathrm{~T}$, a template bearing seven sites (lanes 7-12). In contrast, on $\mathrm{Z}_{1} \mathrm{E} 4 \mathrm{~T}$, a template bearing a single site, although ZEBRA did not stimulate transcription above basal levels at low TFIID concentrations ( 20 or $100 \mathrm{ng}$ ), it generated a moderate effect at the highest level of TFIID (500 ng). The data are tabulated in bar graph form in Figure 5B. The solid black bars indicate the transcription signal ob- 
Figure 5. Synergistic transcription is only observed at limiting TFIID concentrations. (A) Run-off transcription assay showing that high TFIID concentrations reduce synergy. The ${ }^{32} \mathrm{P}$-labeled $\mathrm{Z}_{1} \mathrm{E} 4 \mathrm{~T}$ or $\mathrm{Z}_{7} \mathrm{E} 4 \mathrm{~T}$ templates bearing one or seven ZIIIB sites upstream of the E4 TATA and coding sequence were incubated with ZEBRA, TFIIA, and the indicated amounts of TFIID. After $30 \mathrm{~min}$, heat-treated nuclear extracts and nucleoside triphosphates (NTPs) were added and after $1 \mathrm{hr}$ the reactions were terminated and the products fractionated on a $6 \%$ polyacrylamide/urea gel. The templates and the transcripts are indicated by arrows. $(B) \mathrm{Bar}$ graph comparing transcriptional stimulation and synergy at various TFIID concentrations. The solid and stippled bars indicate the fold-stimulation (activated divided by basal) on templates bearing one and seven sites, respectively. The open bar denotes the hypothetical stimulation on the seven-site template if the transcription was additive with respect to the one-site template. The ratios of the actual stimulation over this hypothetical value are taken as a measure of synergy. $(C)$ A DNase I footprint showing the additive effect of ZEBRA (Z) on the isomerization of the DA complex. Templates bearing one or seven ZIIIB sites upstream of the E4 core promoter were incubated with saturating amounts of ZEBRA and TFIID and assayed by DNase I footprinting. The multiple enhancements, as indicated by arrows, are on average four times stronger on the seven-site template than on a one-site template as quantified by laser densitometry.

served with the template bearing a single site $\left(Z_{1}\right)$, whereas the stippled bars represent the transcription signal from the template containing seven sites $\left\langle Z_{7}\right|$. The open bars represent the signal we would predict if the activators were having a simple additive effect on transcription with respect to the seven-site template. When the observed signal exceeds the predicted signal, tran- scription is considered synergistic and the ratio of the two bars is the fold synergy. We found that under the conditions used here ZEBRA exhibits two- to threefold synergism at the lower concentrations of TFIID, but the synergistic effect diminishes gradually and is not detectable at the highest TFIID concentration.

To rule out the possibility that the loss of synergy at
Figure 6. A model for gene activation by ZEBRA. Multiple ZEBRA molecules (in black), bound upstream of the TATA box and initiation site, synergistically interact with TFIID and TFIIA as denoted by the arrows, resulting in DA complex assembly with a concomitant isomerization, which generates an extended footprint, apparently attributable to TAFs, covering the start site (arrow) and downstream regions. The presence of TFIIB enhances the synergistic action of ZEBRA and the stability of the complex. These complexes then serve as a platform for the entry of holoenzyme (or the other general factors); the start site is melted subsequently to form the open complex before initiation. However, ZEBRA is dispensable for these latter steps; the isomerized DA complex, once formed, is relatively stable in the absence of ZEBRA and able to bind TFIIB and the holoenzyme to generate the final open complex.

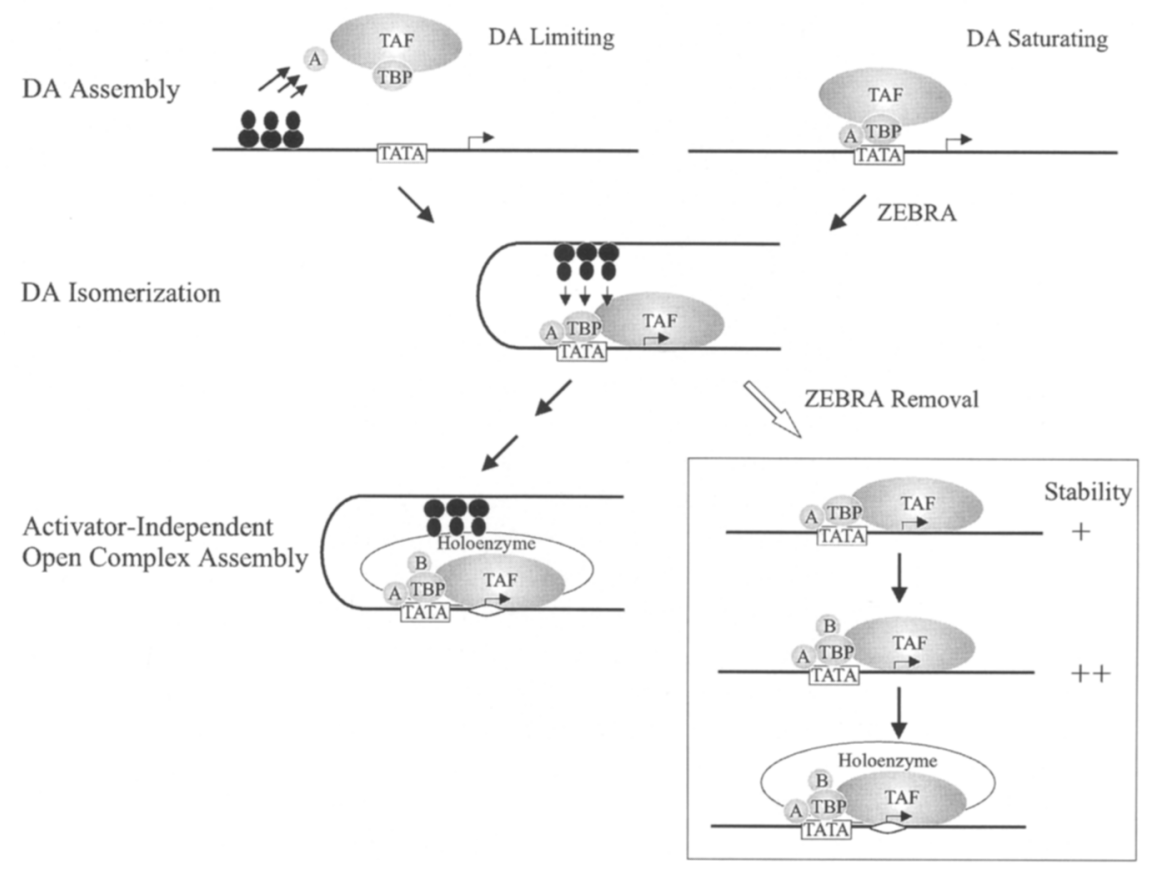


saturating TFIID concentration results from the fact that transcription on the seven-site template has plateaued because transcription factors have become limiting, we repeated the experiment with 10 -fold lower template concentrations and obtained similar results /data not shown). We conclude that synergistic activation by ZEBRA occurs when TFIID is limiting.

Figure 5C shows that the magnitude of the DA isomerization correlates well with the observed transcriptional effects described above. In the presence of saturating DA (lane 3), ZEBRA induces a significant conformational change (lane 4) on a template bearing a single site. By comparing the intensity of the enhanced bands (arrows) using a laser densitometer, we calculated that the isomerization effect observed on seven sites (lane 8) is fourfold greater than that seen with a single site (lane 4), similar in magnitude to the threefold effect seen in the in vitro transcription assay performed under DA saturating conditions. We conclude that when DA is saturating, ZEBRA stimulates DA isomerization and transcription in an additive or nonsynergistic manner.

\section{Discussion}

The model in Figure 6 illustrates our current biochemical view of ZEBRA-mediated gene activation based on the data presented here and in a previous study (Chi et al. 1995). The key features are that both recruitment and isomerization of the DA complex contribute to gene activation by ZEBRA, whereas interaction of ZEBRA with TFIIB further enhances stimulation when TFIID is limiting. This model accommodates many disparate observations in the literature and can provide a framework for understanding the mechanism of transcriptional synergy.

Effects of ZEBRA on the DA complex: recruitment vs. isomerization

By using the same ${ }^{32}$ P-labeled templates for in vitro transcription and DNase I footprinting in a heat-treated nuclear extract, the present work establishes conclusively the DA complex as the major target for ZEBRA in vitro. ZEBRA both recruits and isomerizes the complex probably through direct protein-protein interactions with TAFs and TFIIA. Once isomerized, the complex binds TFIIB and the remaining general factors even after removal of activator. Although the recruitment of the DA complex and its isomerization may be manifestations of the same ZEBRA-DA interactions, we demonstrated that the latter could be induced by a single ZEBRA molecule, whereas the former required multiple contacts and is, thus, the basis for transcriptional synergy in our system. The data confirm the regulatory importance of the conformational changes in the DA complex as exhibited by the extended TFIID footprint first described a decade ago by Roeder and colleagues (Horikoshi et al. $1988 \mathrm{a}, \mathrm{b})$.

The extended footprint per se apparently represents only one indicator of the isomerization event because the ZEBRA-activated DA complex formed on the adenovirus major late promoter, where the extended footprint is constitutive, could also support a detectably higher level of transcription after removal of ZEBRA (data not shown). This observation suggests that other alterations in the DA complex, possibly including the kinase activity of TAF250, may be induced in concert with the extended footprint.

Although ZEBRA has an unusually potent effect on the DA complex, DA is also a critical target for other activators including GAL4-AH and GAL4-VP16. Indeed, GAL4-VP16 stimulates the DA complex assembly and induces its isomerization (White et al. 1992; Chi et al. 1995). Although the GAL4-VP16-isomerized DA complex can support activated transcription after removal of activator (data not shown), it is not as effective in our hands as ZEBRA. However, other studies have confirmed that VP16-induced isomerization does indeed play a key role in activation. For example, in a transcription system reconstituted with crude HeLa TFIID and TFIIA fractions, White and colleagues (1992) have shown that VP16 acts on the preassembled DA complex, the resulting complex being resistant to functional inactivation of GAL4-VP16 with an antibody against its activation domain. Furthermore, our previous kinetic studies showed that preincubation of TFIID and TFIIA with another acidic activator, GAL4-AH, could overcome a ratelimiting step in open complex formation and transcription (Wang et al. 1992a; Chi and Carey 1993). Finally, our data are congruent with the studies of Shykind and colleagues (1995) on the effect of the coactivator HMG2 on DA. With respect to the latter study, although the isomerization did not require HMG2, it may have been stabilized rapidly by the high concentrations of HMG2 in the nuclear extract. Thus, recruitment and isomerization of the DA complex may be a general effect of many activators and coactivators.

Interestingly, several previous reports using reconstituted transcription systems demonstrated that the activator-stimulated $\mathrm{D}$ or DA complex is not sufficient to confer resistance to oligonucleotide challenge (Hai et al. 1988; Lieberman 1994). Perhaps the differences in the stoichiometry of factors may account partially for the discrepancy with our results. Addition of the USA fraction, which has both positive and negative components, into the heat-treated nuclear extract, increased the sensitivity of the ZEBRA-isomerized DA complex to oligonucleotide challenge (data not shown). Thus, the HeLa extracts may normally contain an optimal balance of factors necessary to recognize the isomerized DA complex. Alternatively, HeLa extracts are also a source of the holoenzyme, which may be responsible for their strong stimulatory activity (Maldonado et al. 1996). It is plausible that the recruitment of a holoenzyme to the isomerized DA complex may be a simpler process than the stepwise assembly from free components in a reconstituted system.

\section{Effects of ZEBRA on other transcription factors}

Although TFIIB can associate efficiently with the isomerized DA complex in the absence of ZEBRA, a di- 
rect ZEBRA-TFIIB interaction may be important under physiological conditions because it promotes cooperative assembly of the DAB complex, thus enhancing activation and the degree of synergy. Given the fact that TFIIB is part of the yeast holoenzyme (Koleske and Young 1994), a direct contact could also help mediate entry of the holoenzyme into the complex. Furthermore, activator-induced isomerization of holoenzyme components including TFIIB may be important for its association with the DA complex when the latter is in closed conformation, as discussed in the next section. Finally, direct contacts between ZEBRA and other general transcription factors or the holoenzyme may contribute to multiple rounds of transcription. This is suggested by the fact that removal of ZEBRA after DA isomerization has less of an effect on subsequent open complex formation than on a multiple-round transcription assay /cf. Fig. 2 with Fig. 3). Furthermore, using sarkosyl to inhibit reinitiation, we confirmed that our transcription system supports several rounds of reinitiation and, as expected, single round transcription is less susceptible to oligonucleotide challenge.

\section{Gene activation: recruitment vs. isomerization}

Despite tremendous efforts by the field a unified view of gene activation is still lacking. On the one hand, qualitative alterations in the transcription complex have been proposed to mediate activation including the isomerization of TFIID described here, isomerization of TFIIB (Roberts and Green 1994), and the recently detected kinase activity of TAF250 (Dikstein et al. 1996). In support of such mechanisms, in vivo footprinting of the CYCl (Chen et al. 1994) and heat shock promoters (Giardina and Lis 1995) revealed that TFID is bound constitutively to the TATA box in the absence of inducer. Thus, simple recruitment of TFIID to the promoter is not sufficient for activation. Paradoxically, however, activation can result simply from tethering TBP or GAL11 (Barberis et al. 1995; Chatterjee and Struhl 1995; Xiao et al. 1995), a component of holoenzyme, to the promoter. Furthermore, photofootprints on the GALl and 10 promoter (Selleck and Majors 1987) confirm that TFIID is indeed recruited under physiological conditions. Although the recruitment to GAL1 and GAL10 may be analogous to the situation described herein, the tethering experiments are not easily reconcilable with the present study, which conclusively demonstrated that the binding of TFIID alone is not sufficient for high level transcription. One possible resolution is that a special promoter architecture may suffice to induce TFIID isomerization once it is tethered to the DNA. Alternatively, the closed and isomerized states of TFIID may be in equilibrium and subsequent recruitment of the holoenzyme or TFIIB to bound TFIID could trap the isomerized form in vivo. Furthermore, artificially tethering components of the transcriptional machinery in the absence of activator may cause them to bind more tightly than usual and this might in turn favor the isomerized state of TFIID, or that of holoenzyme components, which bind to TFIID. Fi- nally, we note that although recruitment and isomerization are both essential for activation, the relative contribution of the two events may vary with the promoter type, and both regulatory phenomena can be used to control the patterns of a transcriptional response.

\section{Materials and methods}

\section{Factor purification}

Purification of recombinant ZEBRA, recombinant TFIIA, and hemagglutinin (HA) epitope-tagged TFIID were as described, except for a minor modification in the TFIID purification from the HeLa cell line LTR $\alpha 3$ (Zhou et al. 1992). Specifically, the $1 \mathrm{M}$ $\mathrm{KCl}$ phosphocellulose fraction was first dialyzed in buffer $\mathrm{D}$ containing $0.35 \mathrm{M} \mathrm{KCl}, 1 \mathrm{~mm}$ DTT, and $1 \mathrm{~mm}$ PMSF before immunopurification on a resin containing protein A-Sepharose coupled to the 12CA5 anti-HA monoclonal antibody. The resin was washed twice with buffer $\mathrm{D}$ containing $0.35 \mathrm{M} \mathrm{KCl}$, and twice with buffer D containing $0.2 \mathrm{M} \mathrm{KCl}$, before elution. This step enhances the yield of the TFIID without affecting its ability to generate oligonucleotide-refractory DA complex, as measured by DNase I footprinting or transcription assays (data not shown).

DNase I footprinting, Mg-agarose gel shift, in vitro transcription, and open complex assays

The plasmid $Z_{1}$ or $Z_{7} E 4 T C A T$ (Carey et al. 1992) was digested with EcoRI, ${ }^{32} \mathrm{P}$-end-labeled with polynucleotide kinase and $\left[\gamma^{-32} \mathrm{P}\right] \mathrm{ATP}$ and digested again with HindIII to generate the promoter fragment $Z_{1} E 4 T$ (166 bp) and $Z_{7} E 4 T$ (250 bp) used in footprints shown in Figures 1 and $5 \mathrm{C}$. The corresponding templates used for transcription, open complex, and parallel footprinting assays (Figs. 2, 3, and 5) are identical to $Z_{1,7} E 4 T$, except that they bear additional $250 \mathrm{bp}$ of $\mathrm{E} 4$ coding sequence downstream of the core promoter; these templates were similarly end-labeled and excised (Carey et al. 1992) using EcoRI and HindIII. The promoter fragments $\mathrm{Z}_{1,3,7} \mathrm{M}$ used for $\mathrm{Mg}$-agarose gel mobility shift assays (Fig. 4) were also prepared similarly using $\mathrm{Z}_{1,3,7}$ MCAT (Chi et al. 1995) plasmids. The corresponding transcription template used in Figure 1 bears a $Z_{7} M$ promoter fragment and part of chloramphenicol acetyltransferase (CAT) coding sequence synthesized by PCR using the SP6 promoter primer and a ${ }^{32} \mathrm{P}$-labeled downstream primer targeting the CAT coding region (5'-CTCAAAATGTTCTTTACGATGCCATTGGGA-3').

The binding reactions for DNase I footprinting, $\mathrm{Mg}$-agarose gel shift and transcription were as described previously (Chi et al. 1995). The $13-\mu$ l reaction mixtures contained 6 fmoles of the ${ }^{32} \mathrm{P}$-labeled probe, $10 \mathrm{ng}$ of ZEBRA, $40 \mathrm{ng}$ of TFIIA, and the indicated amounts of TFID in binding buffer [12.5 mM HEPES (pH 7.9), $12.5 \%$ glycerol, $5 \mathrm{~mm} \mathrm{MgCl}_{2}, 70 \mathrm{~mm} \mathrm{KCl}, 0.2 \mathrm{~mm}$ EDTA, $60 \mathrm{~mm} \beta$-mercaptoethanol, $0.5 \mathrm{mg} / \mathrm{ml}$ of BSA, and 30 $\mu \mathrm{g} / \mathrm{ml}$ of poly[d(G-C)]. After a $30-$ to $60-\mathrm{min}$ incubation at $30^{\circ} \mathrm{C}$, the complexes were either subjected directly to footprinting (Figures 1 and $5 \mathrm{C}$ ) or gel mobility shift assays (Fig. 4). Alternatively, $7 \mu \mathrm{l}$ of a mixture containing $40 \mu \mathrm{g}$ of heat-treated HeLa nuclear extract in binding buffer was added to the reaction, and the incubation was continued for 15 min before DNase I analysis. The reactions were terminated by addition of $100 \mu$ l of stop buffer containing $0.4 \mathrm{M} \mathrm{NaOAc}, 0.2 \%$ SDS, $10 \mathrm{~mm}$ EDTA, 50 $\mu \mathrm{g} / \mathrm{ml}$ of yeast tRNA, and $10 \mu \mathrm{g}$ of proteinase K. After a $15-\mathrm{min}$ incubation at $55^{\circ} \mathrm{C}$, the mixtures were extracted with phenolchloroform and the DNA in the aqueous phase was ethanol 
precipitated and resolved on $4 \%$ polyacrylamide $/ 7 \mathrm{M}$ urea sequencing gels. For parallel transcription assays, $1.9-\mu \mathrm{l}$ mixtures containing $0.4 \mu \mathrm{l}$ of $25 \mathrm{~mm}$ each of ATP, GTP, and CTP, $0.4 \mu \mathrm{l}$ of $1 \mathrm{mM}$ UTP, $1 \mu \mathrm{l}$ of $\left[\alpha^{-32} \mathrm{P}\right] \mathrm{UTP}(400 \mathrm{Ci} / \mathrm{mmole}, 10 \mathrm{mCi} / \mathrm{ml})$ and $0.1 \mathrm{ml}$ of RNAsin (Pharmacia) were added in place of DNase I. After $15-20 \mathrm{~min}$ at $30^{\circ} \mathrm{C}$ the transcription reactions were terminated by addition of $100 \mu \mathrm{l}$ stop buffer. The products were purified by phenol-chloroform extraction and resolved subsequently on $6 \%$ polyacrylamide sequencing gels. Heat-treated nuclear extracts were prepared by incubating $300 \mu \mathrm{l}$-aliquots of nuclear extracts at $47^{\circ} \mathrm{C}$ for $15 \mathrm{~min}$ as described (Nakaiima et al. 1988). To prepare TFIID- and TFIIB-depleted nuclear extracts, nuclear extracts were first immunodepleted of TFIIB using affinity-purified antibody as described (Kim and Roeder 1994). The extracts were then subjected to heat treatment.

The open complex assays were essentially the same as above except that $\beta$-mercaptoethanol was replaced by $1 \mathrm{~mm}$ DTT in the binding reactions and that $14 \mu \mathrm{l}$ of heat-treated nuclear extract mixture was added to the binding reactions. These modifications did not affect the transcription outcomes /data not shown). dATP and $\alpha$-amanitin were added to final concentrations of $0.5 \mathrm{~mm}$ and $2 \mathrm{ng} / \mu \mathrm{l}$, respectively. The modified thymidines encompassing the E4 start were detected by primer extension with Taq DNA polymerase as described previously (Chi and Carey 1993; Wang et al. 1992b).

\section{Acknowledgments}

We thank Rod Hori and Dean Tantin for generous sharing of reagents, Sung Pyo for excellent technical assistance, and both Josh Brickman and members of the Carey laboratory for discussion and reading the manuscript. This work was supported by a grant from the American Cancer Society (MV-547).

The publication costs of this article were defrayed in part by payment of page charges. This article must therefore be hereby marked "advertisement" in accordance with 18 USC section 1734 solely to indicate this fact.

\section{References}

Barberis, A., J. Pearlberg, N. Simkovich, S. Farrell, P. Reinagel, C. Bamdad, G. Sigal, and M. Ptashne. 1995. Contact with a component of the polymerase II holoenzyme suffices for gene activation. Cell 81: 359-368.

Carey, M., J. Kolman, D.A. Katz, L. Gradoville, L. Barberis, and G. Miller. 1992. Transcriptional synergy by the Epstein-Barr virus transactivator ZEBRA. J. Virol. 66: 4803-4813.

Carey, M.F. 1995. Transcriptional activation. A holistic view of the complex. Curr. Biol. 5: 1003-1005.

Chao, D.M., E.L. Gadbois, P.J. Murray, S.F. Anderson, M.S. Sonu, J.D. Parvin, and R.A. Young. 1996. A mammalian SRB protein associated with an RNA polymerase II holoenzyme. Nature 380: 82-85.

Chatteriee, S. and K. Struhl. 1995. Connecting a promoterbound protein to TBP bypasses the need for a transcriptional activation domain. Nature 374: 820-822.

Chen, J., M. Ding, and D.S. Peterson. 1994. Binding of TFIID to the Cycl TATA boxes in yeast occurs independently of upstream activating sequences. Proc. Natl. Acad. Sci. 91: 11909-11913.

Chi, T. and M. Carey. 1993. The ZEBRA activation domain: modular organization and mechanism of action. Mol. Cell. Biol. 13: 7045-7055.

Chi, T., P. Lieberman, K. Ellwood, and M. Carey. 1995. A gen- eral mechanism for transcriptional synergy by eukaryotic activators. Nature 377: 254-257.

Choy, B. and M.R. Green. 1993. Eukaryotic activators function during multiple steps of preinitiation complex assembly. Nature 366: 531-536.

Dikstein, R., S. Ruppert, and R. Tjian. 1996. TAFII250 is a bipartite protein kinase that phosphorylates the base transcription factor RAP74. Cell 84: 781-790.

Ge, H. and R.G. Roeder. 1994. Purification, cloning, and characterization of a human coactivator, PC4, that mediates transcriptional activation of class II genes. Cell 78: 513-523.

Giardina, C. and J.T. Lis. 1995. Dynamic protein-DNA architecture of a yeast heat shock promoter. Mol. Cell. Biol. 15: $2737-2744$.

Goodrich, J.A., G. Cutler, and R. Tjian. 1996. Contacts in context: Promoter specificity and macromolecular interactions in transcription. Cell 84: 825-830.

Hai, T.W., M. Horikoshi, R.G. Roeder, and M.R. Green. 1988. Analysis of the role of the transcription factor ATF in the assembly of a functional preinitiation complex. Cell 54: 1043-1051.

Holstege, F.C., P.C. van der Vliet, and H.T. Timmers. 1996. Opening of an RNA polymerase II promoter occurs in two distinct steps and requires the basal transcription factors IIE and IIH. EMBO I. 15: 1666-1677.

Hori, R. and M. Carey. 1994. The role of activators in assembly of RNA polymerase II transcription complexes. Curr. Opin. Genet. Dev. 4: 236-244.

Horikoshi, M., M.F. Carey, H. Kakidani, and R.G. Roeder. 1988a. Mechanism of action of a yeast activator: Direct effect of GAL4 derivatives on mammalian TFIID-promoter interactions. Cell 54: 665-669.

Horikoshi, M., T. Hai, Y.S. Lin, M.R. Green, and R.G. Roeder. 1988b. Transcription factor ATF interacts with the TATA factor to facilitate establishment of a preinitiation complex. Cell 54: 1033-1042.

Kaiser, K., G. Stelzer, and M. Meisterernst. 1995. The coactivator p15 (PC4) initiates transcriptional activation during TFIIA-TFIID-promoter complex formation. EMBO J. 14: 3520-3527.

Kim, T.K. and R.G. Roeder. 1994. Proline-rich activator CTF1 targets the TFIIB assembly step during transcriptional activation. Proc. Natl. Acad. Sci. 91: 4170-4174.

Klages, N. and M. Strubin. 1995. Stimulation of RNA polymerase II transcription initiation by recruitment of TBP in vivo. Nature 374: 822-823.

Kobayashi, N., T.G. Boyer, and A.J. Berk. 1995. A class of activation domains interacts directly with TFIIA and stimulates TFIIA-TFIID-promoter complex assembly. Mol. Cell. Biol. 15: 6465-6473.

Koleske, A.J., and R.A. Young. 1994. An RNA polymerase II holoenzyme responsive to activators [see comments]. $\mathrm{Na}$ ture 368: 466-469.

- 1995. The RNA polymerase II holoenzyme and its implications for gene regulation. Trends Biochem. Sci. 20: 113116.

Lieberman, P. 1994. Identification of functional targets of the Zta transcriptional activator by formation of stable preinitiation complex intermediates. Mol. Cell. Biol. 14: 83658375.

Lieberman, P.M. and A.J. Berk. 1994. A mechanism for TAFs in transcriptional activation: Activation domain enhancement of TFIID-TFIIA-promoter DNA complex formation. Genes \& Dev. 8: 995-1006.

Lin, Y.S. and M.R. Green. 1991. Mechanism of action of an acidic transcriptional activator in vitro. Cell 64: 971-981. 
Lin, Y.S., I. Ha, E. Maldonado, D. Reinberg, and M.R. Green. 1991. Binding of general transcription factor TFIIB to an acidic activating region. Nature 353: $569-571$.

Maldonado, E., R. Shiekhattar, M. Sheldon, H. Cho, R. Drapkin, P. Rickert, E. Lees, C.W. Anderson, S. Linn, and D. Reinberg. 1996. A human RNA polymerase II complex associated with SRB and DNA-repair proteins. Nature 381: 86-89.

Nakajima, N., M. Horikoshi, and R.G. Roeder. 1988. Factors involved in specific transcription by mammalian RNA polymerase II: Purification, genetic specificity, and TATA boxpromoter interactions of TFIID. Mol. Cell. Biol. 8: 40284040.

Ossipow, V., J.P. Tassan, E.A. Nigg, and U. Schibler. 1995. A mammalian RNA polymerase II holoenzyme containing all components required for promoter-specific transcription initiation. Cell 83: 137-146.

Roberts, S.G. and M.R. Green. 1994. Activator-induced conformational change in general transcription factor TFIIB. $\mathrm{Na}$ ture 371: 717-720.

Roberts, S.G., I. Ha, E. Maldonado, D. Reinberg, and M.R. Green. 1993. Interaction between an acidic activator and transcription factor TFIIB is required for transcriptional activation. Nature 363: 741-744.

Roberts, S.G., B. Choy, S.S. Walker, Y.S. Lin, and M.R. Green. 1995. A role for activator-mediated TFIIB recruitment in diverse aspects of transcriptional regulation. Curr. Biol. 5: 508-516.

Sauer, F., S.K. Hansen, and R. Tjian. 1995. Multiple TAFIIs directing synergistic activation of transcription [comment]. Science 270: 1783-1788.

Selleck, S.B. and J. Majors. 1987. Photofootprinting in vivo detects transcription-dent changes in yeast TATA boxes. $\mathrm{Na}$ ture 325: 173-177.

Shykind, B.M., J. Kim, and P.A. Sharp. 1995. Activation of the TFIID-TFIIA complex with HMG-2. Genes \& Dev. 9: 1354 1365.

Stargell, L.A. and K. Struhl. 1995. The TBP-TFIIA interaction in the response to acidic activators in vivo. Science 269: 75-78.

Sundseth, R. and U. Hansen. 1992. Activation of RNA polymerase II transcription by the specific DNA-binding protein LSF. Increased rate of binding of the basal promoter factor TFIIB. J. Biol. Chem. 267: 7845-7855.

Tjian, R. and T. Maniatis. 1994. Transcriptional activation: A complex puzzle with few easy pieces. Cell 77: 5-8.

Wang, W., M. Carey, and J.D. Gralla. 1992a. Polymerase II promoter activation: Closed complex formation and ATPdriven start site opening. Science 255: 450-453.

Wang, W., J.D. Gralla, and M. Carey. 1992b. The acidic activator GAL4-AH can stimulate polymerase II transcription by promoting assembly of a closed complex requiring TFIID and TFIIA. Genes \& Dev. 6: 1716-1727.

White, J., C. Brou, J. Wu, Y. Lutz, V. Moncollin, and P. Chambon. 1992. The acidic transcriptional activator GAL-VP16 acts on preformed template-committed complexes. EMBO $\mathrm{I}$. 11: 2229-2240.

Xiao, H., J.D. Friesen, and J.T. Lis. 1995. Recruiting TATAbinding protein to a promoter: Transcriptional activation without an upstream activator. Mol. Cell. Biol. 15: 57575761.

Zawel, L. and D. Reinberg. 1995. Common themes in assembly and function of eukaryotic transcription complexes. Annu. Rev. Biochem. 64: 533-561.

Zhou, Q., P.M. Lieberman, T.G. Boyer, and A.J. Berk. 1992. Holo-TFIID supports transcriptional stimulation by diverse activators and from a TATA-less promoter. Genes \& Dev. 6: 1964-1974. 


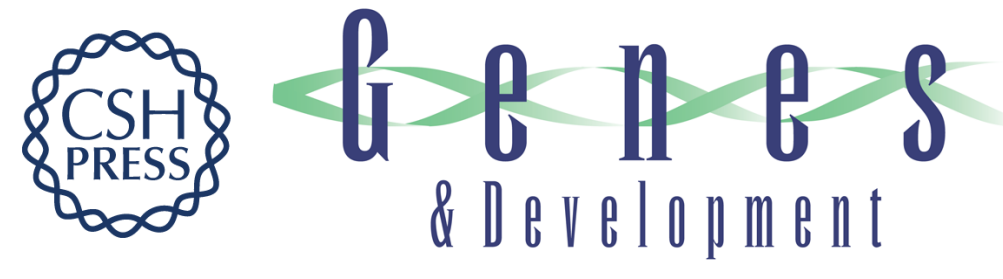

\section{Assembly of the isomerized TFIIA--TFIID--TATA ternary complex is necessary and sufficient for gene activation.}

T Chi and M Carey

Genes Dev. 1996, 10:

Access the most recent version at doi:10.1101/gad.10.20.2540

References

This article cites 46 articles, 16 of which can be accessed free at: http://genesdev.cshlp.org/content/10/20/2540.full.html\#ref-list-1

License

Email Alerting

Receive free email alerts when new articles cite this article - sign up in the box at the top Service right corner of the article or click here.

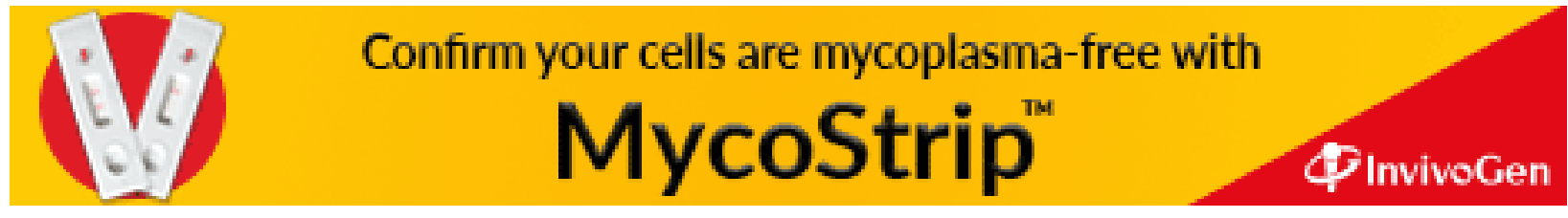

